TITLE:

\title{
A limiting property of the inverse of sampled-data systems on a finite- time interval
}

\author{
$\operatorname{AUTHOR}(S)$ :
}

Sogo, T; Adachi, N

\section{CITATION:}

Sogo, T ...[et al]. A limiting property of the inverse of sampled-data systems on a finitetime interval. IEEE TRANSACTIONS ON AUTOMATIC CONTROL 2001, 46(5): 761-765

\section{ISSUE DATE:}

2001-05

URL:

http://hdl.handle.net/2433/50315

\section{RIGHT:}

(c) 2001 IEEE. Personal use of this material is permitted. However, permission to reprint/republish this material for advertising or promotional purposes or for creating new collective works for resale or redistribution to servers or lists, or to reuse any copyrighted component of this work in other works must be obtained from the IEEE. 


\section{A Limiting Property of the Inverse of Sampled-Data Systems on a Finite-Time Interval}

Takuya Sogo and Norihiko Adachi

\begin{abstract}
If one considers a sampled-data system derived from a continuous-time system with a relative degree of one or two on a finite-time interval, it is not simple to predict the behavior of the output of the inverse of the sampled-data system as the sampling period goes to zero. This is because the number of sample points increases while the zeros of the pulse-transfer function tend to the boundary between the stable and unstable areas. This paper shows that the output of the sampled-data inverse systems converges to the output of the continuous-time inverse systems independently of the stability of zeros.
\end{abstract}

Index Terms-Inverse systems, iterative learning control, limiting zeros, nonminimum phase systems, sampled-data systems.

\section{INTRODUCTION}

Stable inverse systems or stable zeros of transfer functions are often required in many kinds of control problems defined on the infinite time horizon. However, since there is no simple relation between zeros of the pulse-transfer function of sampled-data systems and zeros of the transfer function of continuous-time systems, the behavior of the zeros of sampled-data systems has drawn much attention from researchers. Several approaches to determine the stability of zeros or avoid unstable zeros have been presented [1]-[10]. On the other hand, when control problems are defined on a fixed finite-time interval $\left[0, t_{f}\right]$, one can admit unstable systems unless signals become too large inside $\left[0, t_{f}\right]$. One such finite-time control problems is, for example, iterative learning control which is a trial-based iterative method to improve the transient response on a short time interval [11], [12]. When continuous time systems or their inverse systems are considered on $\left[0, t_{f}\right]$, the peak of the signals is simply determined by the distance between the imaginary axis and poles or zeros, respectively. However, when sampled-data systems with a sampling period $\Delta$ are considered on $\left[0, t_{f}\right]$, the peak of the signals depends on the variable $\Delta$. This relationship is not simple because $\Delta$ changes both the number of sample points inside $\left[0, t_{f}\right]$ and the location of poles and zeros. Furthermore, the zeros as a function of $\Delta$ are much more complicated than the poles. For example, consider the continuous time systems $G_{1}(s)=(s-1) / s^{2}$ and $G_{2}(s)=$ $(2 s-3) /\{(s+1)(s+2)(s+3)\}$. Then, the sampled-data systems derived from each with a sampler and a zero-order hold are $H_{1}(z)=$ $\{(2-\Delta) \Delta(z-(2+\Delta) /(2-\Delta))\} /\left\{2(z-1)^{2}\right\}$ and $H_{2}(z)=$ $f(\Delta)\left(z-q_{1}(\Delta)\right)\left(z-q_{2}(\Delta)\right) /\{(z-\exp (-\Delta))(z-\exp (-2 \Delta))(z-$ $\exp (-3 \Delta))\}$, respectively, where $q_{1}(\Delta)=1+3 \Delta / 2+O\left(\Delta^{2}\right)$ and $q_{2}(\Delta)=-1+5 \Delta / 2+O\left(\Delta^{2}\right)$ [8], [9]. Consider the inverse systems $H_{1}^{-1}(z)$ and $H_{2}^{-1}(z)$ on $\left\{0,1, \ldots, t_{f} / \Delta\right\}$ where $t_{f} / \Delta$ is assumed to be a natural number and give $\left\{y_{d}(0), y_{d}(\Delta), \ldots, y_{d}\left(t_{f}\right)\right\}$ that is the sampled-data of a function $y_{d}(t)$ to $H_{1}^{-1}(z)$ and $H_{2}^{-1}(z)$ as their inputs. Then, the unstable zeros for a small $\Delta$ make the output of the inverse systems increase exponentially. However, it is not easy to determine whether the output of the inverse systems diverges or converges inside $\left[0, t_{f}\right]$ when $\Delta$ goes to zero, because all the zeros tend to points on the unit circle, i.e., the boundary between the stable and unstable areas, while the number of sample points increases inside $\left[0, t_{f}\right]$.

Manuscript received November 29, 1999; revised August 3, 2000 and October 31, 2000. Recommended by Associate Editor T. Chen.

The authors are with the Graduate School of Informatics, Kyoto University, Kyoto 606-8501, Japan (e-mail: sogo@i.kyoto-u.ac.jp).

Publisher Item Identifier S 0018-9286(01)03616-9.
It should be noted that this property of zeros is common for systems whose relative degree is one or two [2], [8], [9]. In this paper, we will discuss such a limiting problem on the fixed time interval for systems with a relative degree of one or two. We will demonstrate that the output of the sampled-data inverse systems converges to the output of the continuous time inverse systems independently of the stability of the zeros when $\Delta$ goes to zero.

\section{Mathematical PRELIMINARIES}

Consider a linear continuous-time single-input-single-output (SISO) system

$$
\begin{aligned}
\frac{d}{d t} x(t) & =A_{c} x(t)+b_{c} u(t) \\
y(t) & =c x(t)
\end{aligned}
$$

where $x \in R^{n}, u \in R$, and $y \in R$ and a sampled-data system derived from (1) with a zero-order hold and a sampler with a sampling period $\Delta$. Then, we have

$$
\begin{aligned}
x((k+1) \Delta) & =A_{\Delta} x(k \Delta)+b_{\Delta} u(k \Delta) \\
y(k \Delta) & =c x(k \Delta)
\end{aligned}
$$

where $A_{\Delta}=\exp A_{c} \Delta$ and $b_{\Delta}=\int_{0}^{\Delta} \exp \left(A_{c} \tau\right) b_{c} d \tau$. Assume that the transfer function $G(s)=c\left(s I-A_{c}\right)^{-1} b_{c}$ is expressed as

$$
G(s)=\frac{K\left(s-\gamma_{1}\right)\left(s-\gamma_{2}\right) \cdots\left(s-\gamma_{m}\right)}{\left(s-p_{1}\right)\left(s-p_{2}\right) \cdots\left(s-p_{n}\right)} .
$$

Then, since there exists a positive constant $\epsilon_{0}$ such that $c b_{\Delta} \neq 0$ for all $\Delta \in\left(0, \epsilon_{0}\right)$, the pulse-transfer function $H(z)=c\left(z I-A_{\Delta}\right)^{-1} b_{\Delta}$ can be expressed as

$$
H(z)=\frac{c b_{\Delta}\left(z-q_{1}(\Delta)\right) \cdots\left(z-q_{n-1}(\Delta)\right)}{\left(z-\exp \left(p_{1} \Delta\right)\right) \cdots\left(z-\exp \left(p_{n} \Delta\right)\right)} .
$$

Next, consider a system (1) with the initial condition $x(0)=0$ on a finite-time interval $\left[0, t_{f}\right]$. Then, the input-output mapping defined by (1) is expressed as $y=S u\left(u, y \in L_{2}\left[0, t_{f}\right]\right)$ where

$$
S u=\int_{0}^{t} c e^{A_{c}(t-\tau)} b_{c} u(\tau) d \tau
$$

Moreover, assume that the sampling period $\Delta$ satisfies $\Delta=t_{f} / N$, where $N$ is a natural number. Then, the input-output relationship on the sample points $\left\{0, \Delta, 2 \Delta, \ldots, t_{f}-\Delta, t_{f}\right\}$ is

$$
w_{\Delta}=\Gamma_{\Delta} v_{\Delta}
$$

where

$$
\begin{aligned}
v_{\Delta} & =\left[\begin{array}{llll}
u(0) & u(\Delta) & \cdots & u\left(t_{f}\right)
\end{array}\right]^{\mathrm{T}} \\
w_{\Delta} & =\left[\begin{array}{llll}
y(0) & y(\Delta) & \cdots & y\left(t_{f}\right)
\end{array}\right]^{\mathrm{T}} \\
\Gamma_{\Delta} & =\left[\begin{array}{cccc}
0 & & & 0 \\
m_{\Delta}(1) & & & \\
\vdots & \ddots & & \\
m_{\Delta}(N) & \cdots & m_{\Delta}(1) & 0
\end{array}\right]
\end{aligned}
$$

where the Markov parameter $m_{\Delta}(i)(i=1, \ldots, N)$ is defined as $m_{\Delta}(i)=c A_{\Delta}^{i-1} b_{\Delta}$. 
Note that $c b_{\Delta} \neq 0$ for almost all $\Delta>0$. Then, we have the inverse system

$$
\begin{aligned}
x((k+1) \Delta)= & \left\{A_{\Delta}-b_{\Delta}\left(c b_{\Delta}\right)^{-1} c A_{\Delta}\right\} x(k \Delta) \\
& +b_{\Delta}\left(c b_{\Delta}\right)^{-1} y((k+1) \Delta) \\
u(k \Delta)= & -\left(c b_{\Delta}\right)^{-1} c A_{\Delta} x(k \Delta) \\
& +\left(c b_{\Delta}\right)^{-1} y((k+1) \Delta) \\
(k= & 0, \ldots, N-1) .
\end{aligned}
$$

By letting $u(N \Delta)=0$, (10) defines a mapping from $w_{\Delta}$ to $v_{\Delta}$. We can denote the mapping as $\Gamma_{\Delta}^{+}$, the Moore-Penrose generalized inverse $\Gamma_{\Delta}^{+}$of $\Gamma_{\Delta}$, which is the mapping from $w_{\Delta}$ to the minimizer of $\left(w_{\Delta}-\right.$ $\left.\Gamma_{\Delta} v_{\Delta}\right)^{\mathrm{T}}\left(w_{\Delta}-\Gamma_{\Delta} v_{\Delta}\right)$ with the minimum norm.

In the following discussions, we will use the sampling operator $\sigma_{\Delta}$ : $L_{2}\left[0, t_{f}\right] \rightarrow R^{N+1}$ and the zero-order hold operator $\theta_{\Delta}: R^{N+1} \rightarrow$ $L_{2}\left[0, t_{f}\right]$, defined as follows:

$$
\begin{aligned}
\sigma_{\Delta}[u] & =\left[u(0) u(\Delta) \cdots u((N-1) \Delta) u\left(t_{f}\right)\right]^{\mathrm{T}} \\
{\left[\theta_{\Delta} v\right](t) } & =\left\{\begin{array}{ll}
v(k) & \text { if } \quad t \in[(k-1) \Delta, k \Delta] \\
v\left(\frac{{ }^{t}}{\Delta}+1\right) & \text { if } t=t_{f}
\end{array} .\right.
\end{aligned}
$$

We define the following notations: $\|u\|_{\infty}=\sup \left\{|u(t)| ; t \in\left[0, t_{f}\right)\right\}$, $|v|_{\infty}^{N}=\sup \left\{\left|v_{i}\right| ; i=1,2, \ldots, N\left(=t_{f} / \Delta\right)\right\}, C^{k}\left[0, t_{f}\right] \mathrm{de}-$ notes set of $k$-th continuously differentiable functions on $\left[0, t_{f}\right]$. Note that $\Gamma_{\Delta} v=\sigma_{\Delta} S \theta_{\Delta} v$ and if $u^{*} \in C^{k}\left[0, t_{f}\right](k \geq 1)$ then $\lim _{\Delta \rightarrow 0}\left\|\theta_{\Delta} \sigma_{\Delta} u^{*}-u^{*}\right\|_{\infty}=0$.

In the following sections, we consider the inverse discrete-time system with sampled data of a fixed function $y^{*}$ defined in $\left[0, t_{f}\right]$ as the input, i.e., $\Gamma_{\Delta}^{+} \sigma_{\Delta} y^{*}$ or $(10)$ whose $y((k+1) \Delta)$ is substituted with $y^{*}((k+1) \Delta)$.

\section{The MAIN RESUlTS}

In this section, we present the limit of $\Gamma_{\Delta}^{+} \sigma_{\Delta} y^{*}$ as $\Delta \rightarrow 0$.

Theorem 1: Assume that $n-m=1$ or 2 and there exists $u^{*} \in$ $C^{n-m-1}\left[0, t_{f}\right]$ such that $y^{*}=S u^{*}$. Then

$$
\left\|\theta_{\Delta} \Gamma_{\Delta}^{+} \sigma_{\Delta} y^{*}-u^{*}\right\|_{\infty} \rightarrow 0
$$

as $\Delta \rightarrow 0$.

Remark 1: The convergence (13) is presented only on $\left[0, t_{f}\right)$, while the function $\theta_{\Delta} \Gamma_{\Delta}^{+} \sigma_{\Delta} y^{*}-u^{*}$ is defined on $\left[0, t_{f}\right]$. However, this is the best result because from the definition of $\Gamma_{\Delta}^{+}$we have $\left[\theta_{\Delta} \Gamma_{\Delta}^{+} \sigma_{\Delta} y^{*}-\right.$ $\left.u^{*}\right]\left(t_{f}\right) \equiv u^{*}\left(t_{f}\right)$, which is independent of $\Delta$.

It should be noted that the result of Theorem 1 is independent of the stability of the zeros of $H(z) ; \Gamma_{\Delta}^{+} \sigma_{\Delta} y^{*}$ converges even if some zeros go to the unit circle from the outside.

If a function $y^{*}(t)\left(t \in\left[0, t_{f}\right]\right)$ satisfies the assumption in Theorem $1, u^{*}$ can be obtained such that $y^{*}=S u^{*}$ by using the following continuous-time inverse system of (1)

$$
\begin{aligned}
\frac{d}{d t} x(t) & =\left\{A_{c}+b_{c} F^{-1} c A_{c}\right\} x(t)+b_{c} F^{-1}\left(\frac{d}{d t}\right)^{n-m} y(t) \\
u(t) & =F^{-1} c A_{c}^{n-m} x(t)+F^{-1}\left(\frac{d}{d t}\right)^{n-m} y(t)
\end{aligned}
$$

where $F=c A_{c}^{n-m-1} b_{c}$ and $x(0)=0$. Let $S^{-1}$ be the input-output mapping of (14) on $\left[0, t_{f}\right]$. Then, $u^{*}=S^{-1} y^{*} ;(13)$ is equivalent to $\left\|\theta_{\Delta} \Gamma_{\Delta}^{+} \sigma_{\Delta} y^{*}-S^{-1} y^{*}\right\|_{\infty} \rightarrow 0$. This means that the discrete-time inverse system (10) converges to the continuous-time inverse system (14) as $\Delta \rightarrow 0$.
The proof of Theorem 1 will be established in the following sequence of lemmas ${ }^{1}$; all assumptions in Theorem 1 will be preserved. Since we have

$$
\begin{aligned}
\left\|\theta_{\Delta} \Gamma_{\Delta}^{+} \sigma_{\Delta} y^{*}-u^{*}\right\|_{\infty} \leq & \left|\Gamma_{\Delta}^{+} \sigma_{\Delta} y^{*}-\sigma_{\Delta} u^{*}\right|_{\infty}^{N} \\
& +\left\|\theta_{\Delta} \sigma_{\Delta} u^{*}-u^{*}\right\|_{\infty} \\
= & \left|\Gamma_{\Delta}^{+}\left(\sigma_{\Delta} S u^{*}-\sigma_{\Delta} S \theta_{\Delta} \sigma_{\Delta} u^{*}\right)\right|_{\infty}^{N} \\
& +\left\|\theta_{\Delta} \sigma_{\Delta} u^{*}-u^{*}\right\|_{\infty} .
\end{aligned}
$$

What we have to show is

$$
\left|\Gamma_{\Delta}^{+}\left(\sigma_{\Delta} S u^{*}-\sigma_{\Delta} S \theta_{\Delta} \sigma_{\Delta} u^{*}\right)\right|_{\infty}^{N} \rightarrow 0
$$

as $\Delta \rightarrow 0$

First, we consider the case of $n-m=1$ and $N \times N$ matrix $\Lambda_{1 \Delta}$ such that $\Gamma_{\Delta}^{+}(N, N+1)=\Lambda_{1 \Delta} \Lambda_{2 \Delta}$ where $\Gamma_{\Delta}^{+}(N, N+1)$ indicates the matrix $\Gamma_{\Delta}^{+}$without its $N+1$-th row; $\Lambda_{2 \Delta}$ is an $N \times(N+1)$ matrix defined as

$$
\Lambda_{2 \Delta}=\frac{1}{\Delta}\left[\begin{array}{cccc}
-1 & 1 & & 0 \\
& \ddots & \ddots & \\
0 & & -1 & 1
\end{array}\right] \text {. }
$$

Consider the inverse system (10) on the infinite interval and the pulse-transfer function $H(z)^{-1}$. Then, we can see that the decomposition of $\Gamma_{\Delta}^{+}(N, N+1)$ given above corresponds to $H(z)^{-1}=H_{1}(z)^{-1} H_{2}(z)^{-1}$ where

$$
\begin{aligned}
& H_{1}(z)^{-1}=\frac{\Delta\left(z-\exp \left(p_{1} \Delta\right)\right) \cdots\left(z-\exp \left(p_{n} \Delta\right)\right)}{c b_{\Delta}(z-1)\left(z-q_{1}(\Delta)\right) \cdots\left(z-q_{n-1}(\Delta)\right)} \\
& H_{2}(z)^{-1}=\frac{(z-1)}{\Delta} .
\end{aligned}
$$

Lemma 1: Assume $n-m=1$ and consider the $N \times N$ matrix $\Lambda_{1 \Delta}$ defined as

$$
\Lambda_{1 \Delta}=\left[\begin{array}{ccc}
m_{1 \Delta}(0) & & 0 \\
\vdots & \ddots & \\
m_{1 \Delta}(N-1) & \cdots & m_{1 \Delta}(0)
\end{array}\right]
$$

where Markov parameters $m_{1 \Delta}(i)(i=0, \ldots, N-1)$ are defined as $m_{1 \Delta}(i)=1 / 2 \pi j \oint_{C} H_{1}(z)^{-1} z^{i-1} d z(C$ : a simple path enclosing all poles) Then, sup $\left\{\left|\Lambda_{1 \Delta} v\right|_{\infty}^{N} /|v|_{\infty}^{N} ; v \in R^{N}\right\}<+\infty$ for $\Delta \in\left(0, \epsilon_{0}\right)$.

Proof: See Appendix.

Lemma 2: Assume $n-m=1$. Then, $\mid \Lambda_{2 \Delta}\left(\sigma_{\Delta} S u^{*}-\right.$ $\left.\sigma_{\Delta} S \theta_{\Delta} \sigma_{\Delta} u^{*}\right)\left.\right|_{\infty} ^{N} \rightarrow 0$ as $\Delta \rightarrow 0$.

Proof: See Appendix.

Next, we consider the case of $n-m=2$ and $N \times N$ matrix $\Lambda_{3 \Delta}$ such that $\Gamma_{\Delta}^{+}(N, N+1)=\Lambda_{3 \Delta} \Lambda_{4 \Delta} \Lambda_{5 \Delta}$ where $\Lambda_{4 \Delta}$ is an $N \times(N-1)$ matrix defined as

$$
\Lambda_{4 \Delta}=\left[\begin{array}{cccc}
0 & & & 0 \\
1 & 0 & & \\
-1 & 1 & \ddots & \\
\vdots & \ddots & \ddots & 0 \\
(-1)^{N} & \cdots & -1 & 1
\end{array}\right]
$$

\footnotetext{
${ }^{1}$ Theorem for the case of $n-m=1$ has been proved in [13], [14]; the approach was based only on a state-space representation. In this paper, we present a refined proof which uses an asymptotic property of zeros of the pulse transfer function.
} 

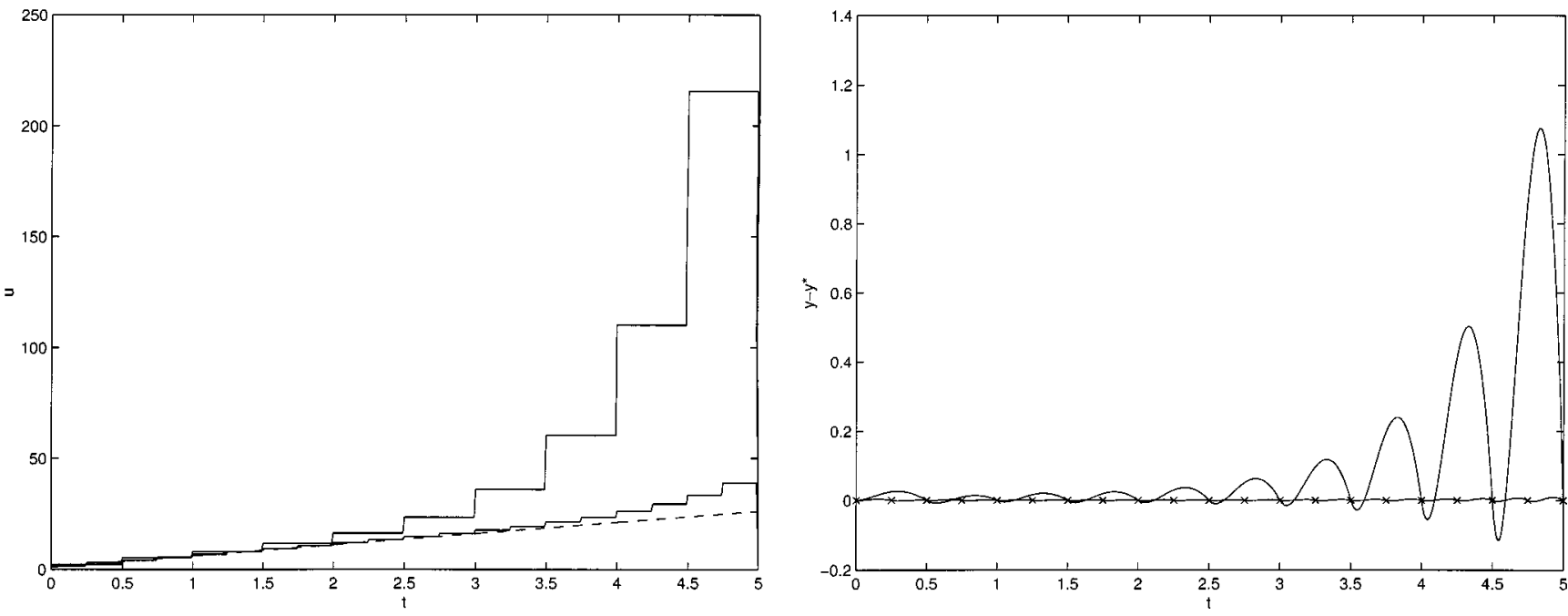

Fig. 1. Left: $u=\theta_{\Delta} \Gamma_{\Delta}^{+} \sigma_{\Delta} y^{*}$ with $u^{*}$. Right: $y-y^{*}=S \theta_{\Delta} \Gamma_{\Delta}^{+} \sigma_{\Delta} y^{*}-y^{*}$.

and $\Lambda_{5 \Delta}$ is an $(N-1) \times(N+1)$ matrix defined as

$$
\Lambda_{5 \Delta}=\frac{1}{\Delta^{2}}\left[\begin{array}{ccccc}
1 & -2 & 1 & & 0 \\
& \ddots & \ddots & \ddots & \\
0 & & 1 & -2 & 1
\end{array}\right] .
$$

We can see that the decomposition of $\Gamma_{\Delta}^{+}(N, N+1)$ given above corresponds to $H(z)^{-1}=H_{3}(z)^{-1} H_{4}(z)^{-1} H_{5}(z)^{-1}$ where

$$
H_{3}(z)^{-1}=\frac{\Delta^{2}(z+1)\left(z-\exp \left(p_{1} \Delta\right)\right) \cdots\left(z-\exp \left(p_{n} \Delta\right)\right)}{c b_{\Delta}(z-1)^{2}\left(z-q_{1}(\Delta)\right) \cdots\left(z-q_{n-1}(\Delta)\right)},
$$

$H_{4}(z)^{-1}=1 /(z+1)$ and $H_{5}(z)^{-1}=(z-1)^{2} / \Delta^{2}$.

Lemma 3: Assume $n-m=2$ and consider the $N \times N$ matrix $\Lambda_{3 \Delta}$ defined as

$$
\Lambda_{3 \Delta}=\left[\begin{array}{ccc}
m_{3 \Delta}(0) & & 0 \\
\vdots & \ddots & \\
m_{3 \Delta}(N-1) & \cdots & m_{3 \Delta}(0)
\end{array}\right]
$$

where Markov parameters $m_{3 \Delta}(i)(i=0, \ldots, N-1)$ are defined as $m_{3 \Delta}(i)=(1 / 2 \pi j) \oint_{C} H_{3}(z)^{-1} z^{i-1} d z(C$ : a simple path enclosing all poles). Then, $\sup \left\{\left|\Lambda_{3 \Delta} v\right|_{\infty}^{N} /|v|_{\infty}^{N} ; v \in R^{N}\right\}<+\infty$ for $\Delta \in$ $\left(0, \epsilon_{0}\right)$.

Proof: See Appendix.

Lemma 4: Assume $n-m=2$. Then, $\mid \Lambda_{4 \Delta} \Lambda_{5 \Delta}\left(\sigma_{\Delta} S u^{*}-\right.$ $\left.\sigma_{\Delta} S \theta_{\Delta} \sigma_{\Delta} u^{*}\right)\left.\right|_{\infty} ^{N} \rightarrow 0$ as $\Delta \rightarrow 0$.

Proof: See Appendix.

We are now ready to establish Theorem 1 . We have seen that $\Gamma_{\Delta}^{+}(N, N+1)=\Lambda_{1 \Delta} \Lambda_{2 \Delta}$ or $\Lambda_{3 \Delta} \Lambda_{4 \Delta} \Lambda_{5 \Delta}$. If $n-m=1$ or 2 , we can obtain (16) from Lemma 2 with Lemma 1, or Lemma 4 with Lemma 3, respectively.

\section{NumERICAL EXAMPLES AND INTERPRETATION OF THE MAIN RESULT}

Consider $G_{2}(s)$ given in Section I on the finite-time interval $[0,5]$ with $u^{*}(t)=5 t+1$ and $y^{*}=S u^{*}$. Then, the pulse transfer function $H_{2}(z)$ has the unstable zero $q_{1}(\Delta)$ for a small sampling period as shown in Section I. Fig. 1 shows $u=\theta_{\Delta} \Gamma_{\Delta}^{+} \sigma_{\Delta} y^{*}$ with $u^{*}$ and $y-y^{*}=S \theta_{\Delta} \Gamma_{\Delta}^{+} \sigma_{\Delta} y^{*}-y^{*}$ for $\Delta=0.5$ and 0.25 . When $\Delta=0.5$, the unstable zero makes the signal $u$ very large at the right end of the time interval; this causes intersample ripples as shown in the right figure.
However, when $\Delta$ is shrunk to 0.25 , we can observe that the signal $u$ is near the ideal input $u^{*}$ and therefore the intersample ripples are eliminated.

The main result shown in this paper implies that the solution of an optimal control problem minimizing $\int_{0}^{t} f\left\{y(t)-y^{*}(t)\right\}^{2} d t$ or the inverse problem finding $u(t)$ such that $y^{*}=S u$ can be approximated by the solution of the finite-dimensional optimal control problem minimizing $\left|\Gamma_{\Delta} v-\sigma_{\Delta} y^{*}\right|^{2}$, namely $\Gamma_{\Delta}^{+} \sigma_{\Delta} y^{*}$ when the relative degree is 1 or 2. This property is favorable for iterative learning control [11], which is a method to realize precise output tracking on $\left[0, t_{f}\right]$ by repetitive improvement based on experimental input-output data; inter-sample residuals of the output can be reduced simply by shrinking the sampling period $\Delta$ as far as precise output tracking is achieved only on the sample points.

\section{CONCLUding REMARKS}

We demonstrated that when the relative degree is one or two, the inverse of sampled-data systems approximates to the inverse of continuous-time systems independently of the stability of the zeros. It should be noted that such a property is uncommon for a relative degree greater than two, because there is at least one zero that converges to a point exterior to the unit circle [2].

\section{APPENDIX I}

\section{PROOF OF LEMMA 1}

Since the first equation shown at the bottom of the next page holds true, the system $\left(\bar{A}_{\Delta}, \bar{b}_{\Delta}, \bar{c}_{\Delta}, \Delta / c b_{\Delta}\right)$ is converted to the controllable canonical form.

$$
\left(\tilde{A}_{\Delta},\left[\begin{array}{c}
r_{0}(\Delta) \\
\vdots \\
r_{n}(\Delta)
\end{array}\right],\left[\begin{array}{llll}
0 & \cdots & 0 & \frac{\Delta}{c b_{\Delta}}
\end{array}\right], \frac{\Delta}{c b_{\Delta}}\right)
$$

where $\left|\tilde{A}_{\Delta}\right|=\max \left\{1,\left|q_{1}(\Delta)\right|, \ldots,\left|q_{n-1}(\Delta)\right|\right\}$. Since we can see $\left|r_{j}(\Delta)\right| / \Delta \leq+\infty(j=0, \ldots, n)$, we have

$$
\left|\left[\begin{array}{c}
r_{0}(\Delta) \\
\vdots \\
r_{n}(\Delta)
\end{array}\right]\right| \leq \bar{M}_{1} \Delta
$$

where $\bar{M}_{1}$ is a positive constant; $|\cdot|$ indicates the Euclidean norm and its induced norm. From the Taylor expansion of the intrinsic zero, namely $q_{i}(\Delta)=1+\gamma_{i} \Delta+O\left(\Delta^{2}\right)[8]$, we have $\left|\tilde{A}_{\Delta}\right| \leq 1+\bar{M}_{2} \Delta \leq e^{\bar{M}_{2} \Delta}$ 
for $\Delta \in\left(0, t_{f}\right)$, where $\bar{M}_{2}$ is a positive constant. Note that there exists a positive constant $\bar{M}_{3}>0$ such that $\left|\Delta / c b_{\Delta}\right|<\bar{M}_{3}$ for almost all $\Delta>0$. Then, we have $\left[\Lambda_{1 \Delta} v\right]_{1} \leq \bar{M}_{3}\left|v_{1}\right|$ and

$$
\begin{aligned}
{\left[\Lambda_{1 \Delta} v\right]_{k} \leq } & \frac{\bar{M}_{1} \bar{M}_{3}}{\bar{M}_{2}}\left(e^{(k-1) \bar{M}_{2} \Delta}-1\right) \\
& \times \max \left\{\left|v_{j}\right| ; j=1, \ldots, k-1\right\}+\bar{M}_{3}\left|v_{k}\right|
\end{aligned}
$$

for $k=2, \ldots, N$. Those inequalities imply that $\sup \left\{\left|\Lambda_{1 \Delta} v\right|_{\infty}^{N} /|v|_{\infty}^{N} ; v \in R^{N}\right\} \quad<\quad+\infty$ because $e^{(k-1) \bar{M}_{2} \Delta}-1 \leq e^{(N-1) \bar{M}_{2} \Delta}-1<e^{T \bar{M}_{2}}-1$.

\section{APPENDIX II}

PROOF OF LEMMA 2

Note

$[S u](t)=\int_{0}^{t}\left\{c A_{c} \int_{0}^{\tau} e^{A_{c}(\tau-\sigma)} b_{c} u(\sigma) d \sigma+c b_{c} u(\tau)\right\} d \tau$. Then, we have the second equation shown at the bottom of the page, for $k=0,1, \ldots, N-1$, where $\xi_{k} \in[k \Delta,(k+1) \Delta]$. Moreover, we have

$$
\begin{aligned}
& \left|\left[\Lambda_{2 \Delta}\left(\sigma_{\Delta} S\left(u^{*}-\theta_{\Delta} \sigma_{\Delta} u^{*}\right)\right)\right](k)\right| \\
& \quad \leq \sup _{t \in\left[0, t_{f}\right]}\left|c A_{c} \int_{0}^{t} e^{A_{c}(t-\sigma)} b_{c} d \sigma+c b_{c}\right| \\
& \quad \times\left\|u^{*}-\theta_{\Delta} \sigma_{\Delta} u^{*}\right\|_{\infty}^{\prime}
\end{aligned}
$$

where $\|u\|_{\infty}^{\prime}=\sup \left\{|u(t)| ; t \in\left[0, t_{f}\right]\right\}$. Since $\left\|u^{*}-\theta_{\Delta} \sigma_{\Delta} u^{*}\right\|_{\infty}^{\prime} \rightarrow$ 0 as $\Delta \rightarrow 0$, we have $\left|\Lambda_{2 \Delta}\left(\sigma_{\Delta} S u^{*}-\sigma_{\Delta} S \theta_{\Delta} \sigma_{\Delta} u^{*}\right)\right|_{\infty}^{N} \rightarrow 0$.
APPENDIX III

PROOF OF LEMMA 3

Since we have $q_{i}(\Delta)=1+\gamma_{i} \Delta+O\left(\Delta^{2}\right)(i=1, \ldots, n-2)$ [15] for the intrinsic zeros and $q_{n-1}(\Delta)=-1+\left(\sum_{i=1}^{m} \gamma_{i}-\sum_{i=1}^{n} p_{i}\right) \Delta / 3+$ $O\left(\Delta^{2}\right)$ for the discretization zero [9], there exists a constant $M_{4}$ such that $\max \left\{1,\left|q_{1}(\Delta)\right|, \ldots,\left|q_{n-1}(\Delta)\right|\right\} \leq e^{M_{4} \Delta}$ for $\Delta \in\left(0, t_{f}\right)$. Therefore, we can see that $\sup \left\{\left|\Lambda_{3} \Delta\right|_{\infty}^{N} /|v|_{\infty}^{N} ; v \in R^{N}\right\}<+\infty$ in the same manner as the proof of Lemma 1 .

\section{APPENDIX IV}

\section{PROOF OF LEMMA 4}

Consider an $N-1\left(t_{f} / \Delta-1\right)$-dimensional vector $w_{\Delta}$ which satisfies $\left|w_{\Delta}\right|_{\infty}^{N-1}<+\infty$ and $\left|v_{\Delta}\right|_{\infty}^{N-2} \rightarrow 0$ as $\Delta \rightarrow 0$, where $v_{\Delta}=\left[w_{\Delta}(1)-w_{\Delta}(2) \cdots w_{\Delta}(N-2)-w_{\Delta}(N-1)\right]^{\mathrm{T}}$. Then, $\left|\Lambda_{4 \Delta} w_{\Delta} \Delta\right|_{\infty}^{N}$ tends to 0 as $\Delta \rightarrow 0$. This is because we have the third equation, shown at the bottom of the page, which implies the fourth equation shown at the bottom of the previous page.

To establish the lemma, we will show that $\Lambda_{5 \Delta} \sigma_{\Delta} S \eta_{\Delta}$, where $\eta_{\Delta}=$ $u^{*}-\theta_{\Delta} \sigma_{\Delta} u^{*}$ has the same limiting property as $w_{\Delta} \Delta$ given above. Let $\zeta_{\Delta}=S \eta_{\Delta}$. Then

$$
\begin{aligned}
\zeta_{\Delta}^{(2)}(t)=\frac{d^{2}}{d t^{2}} S \eta_{\Delta}= & c A_{c}^{2} \int_{0}^{t} e^{A_{c}(t-\tau)} b_{c} \eta_{\Delta}(\tau) d \tau \\
& +c A_{c} b_{c} \eta_{\Delta}(t) \\
\zeta_{\Delta}^{(3)}(t)=\frac{d^{3}}{d t^{3}} S \eta_{\Delta}= & c A_{c}^{3} \int_{0}^{t} e^{A_{c}(t-\tau)} b_{c} \eta_{\Delta}(\tau) d \tau \\
& +c A_{c}^{2} b_{c} \eta_{\Delta}(t)+c A_{c} b_{c} \frac{d}{d t} u^{*}(t) .
\end{aligned}
$$

$$
H_{1}^{-1}(z)=\frac{\Delta}{c b_{\Delta}}\left\{1+\frac{r_{0}(\Delta) z^{n-1}+r_{1}(\Delta) z^{n-2}+\cdots+r_{n}(\Delta)}{(z-1)\left(z-q_{1}(\Delta)\right) \cdots\left(z-q_{n-1}(\Delta)\right)}\right\}
$$

$$
\begin{aligned}
& {\left[\Lambda_{2 \Delta}\left(\sigma_{\Delta} S\left(u^{*}-\theta_{\Delta} \sigma_{\Delta} u^{*}\right)\right)\right](k)} \\
& \quad=\frac{\left[S\left(u^{*}-\theta_{\Delta} \sigma_{\Delta} u^{*}\right)\right]((k+1) \Delta)-\left[S\left(u^{*}-\theta_{\Delta} \sigma_{\Delta} u^{*}\right)\right](k \Delta)}{\Delta} \\
& \quad=c A_{c} \int_{0}^{\xi_{k}} e^{A_{c}\left(\xi_{k}-\sigma\right)} b_{c}\left(u^{*}-\theta_{\Delta} \sigma_{\Delta} u^{*}\right) d \sigma+c b_{c}\left(u^{*}\left(\xi_{k}\right)-u^{*}(k \Delta)\right)
\end{aligned}
$$

$$
\left[\Lambda_{4 \Delta} w_{\Delta} \Delta\right](k)= \begin{cases}0 & k=1 \\ w_{\Delta}(1) \Delta & k=2 \\ (-1)^{k} \sum_{i=1}^{(k-1) / 2} \Delta\left\{w_{\Delta}(i)-w_{\Delta}(i+1)\right\} & k=3,5, \ldots \\ w_{\Delta}(k-1) \Delta & \\ +(-1)^{k} \sum_{i=1}^{k / 2} \Delta\left\{w_{\Delta}(i)-w_{\Delta}(i+1)\right\} & k=4,6, \ldots\end{cases}
$$

$$
\begin{array}{r}
\left|\left[\Lambda_{4 \Delta} w_{\Delta} \Delta\right](k)\right| \leq \max \left\{\left|w_{\Delta}(1)\right| \Delta, \frac{\left(t_{f} / \Delta-1\right)}{2} \Delta\left|v_{\Delta}\right|_{\infty}^{N-2},\right. \\
\left.\left|w_{\Delta}(k-1)\right| \Delta+\left(\frac{t_{f}}{\Delta}\right) \Delta\left|v_{\Delta}\right|_{\infty}^{N-2}\right\} .
\end{array}
$$




$$
\begin{aligned}
{\left[\Lambda_{5 \Delta} \sigma_{\Delta} S \eta_{\Delta}\right](k) } & =\frac{\zeta_{\Delta}((k-1) \Delta)-2 \zeta_{\Delta}(k \Delta)+\zeta_{\Delta}((k+1) \Delta)}{\Delta^{2}} \\
& =\zeta_{\Delta}^{(2)}(k \Delta)+\frac{\zeta_{\Delta}^{(3)}\left(\left(k+\psi_{k}^{\Delta}\right) \Delta\right) \Delta}{6}-\frac{\zeta_{\Delta}^{(3)}\left(\left(k-\phi_{k}^{\Delta}\right) \Delta\right) \Delta}{6}
\end{aligned}
$$

By using the Taylor expansion of $\zeta_{\Delta}((k-1) \Delta)$ and $\zeta_{\Delta}((k+1) \Delta)$, we have the equation shown at the top of the page, where $k=1,2, \ldots, N-1$ and $\psi_{k}^{\Delta}, \phi_{k}^{\Delta} \in[0,1]$. Since $\eta_{\Delta}(k \Delta)=$ $\eta_{\Delta}((k+1) \Delta)=0$ and $\left\|\eta_{\Delta}\right\|_{\infty}^{\prime} / \Delta \leq\left\|(d / d t) u^{*}\right\|_{\infty}^{\prime}$, we have

$$
\left|\frac{\zeta_{\Delta}^{(2)}(k \Delta)}{\Delta}\right| \leq \sup _{t \in\left[0, t_{f}\right]}\left|c A_{c}^{2} \int_{0}^{t} e^{A_{c}(t-\tau)} b_{c} d \tau\right|\left\|\frac{d}{d t} u^{*}\right\|_{\infty}^{\prime}
$$

and by the mean-value theorem

$$
\begin{aligned}
& \left|\frac{\zeta_{\Delta}^{(2)}((k+1) \Delta)}{\Delta}-\frac{\zeta_{\Delta}^{(2)}(k \Delta)}{\Delta}\right| \\
& =\mid c A_{c}^{3} \int_{0}^{\left(k+\theta_{k}^{\Delta}\right) \Delta} e^{A_{c}\left(\left(k+\theta_{k}^{\Delta}\right) \Delta-\tau\right)} b_{c} \eta_{\Delta}(\tau) d \tau \\
& +c A_{c}^{2} b_{c} \eta_{\Delta}\left(\left(k+\theta_{k}^{\Delta}\right) \Delta\right) \\
& \leq \sup _{t \in\left[0, t_{f}\right]}\left|c A_{c}^{3} \int_{0}^{t} e^{A_{c}(t-\tau)} b_{c} d \tau+c A_{c}^{2} b_{c}\right|\left\|\eta_{\Delta}\right\|_{\infty}^{\prime}
\end{aligned}
$$

where $\theta_{k}^{\Delta} \in[0,1]$. Since $\left\|(d / d t) u^{*}\right\|_{\infty}^{\prime}$ is bounded and $\left\|\eta_{\Delta}\right\|_{\infty}^{\prime}$ tends to 0 , we can see that $\left[\zeta_{\Delta}^{(2)}(\Delta) \cdots \zeta_{\Delta}^{(2)}((N-1) \Delta)\right]$ has the same limiting property as $w_{\Delta} \Delta$. Moreover, we have

$$
\begin{aligned}
& \left|\zeta_{\Delta}^{(3)}\left(\left(k+\psi_{k}^{\Delta}\right) \Delta\right)\right| \\
& \quad \leq \sup _{t \in\left[0, t_{f}\right]}\left|c A_{c}^{3} \int_{0}^{t} e^{A_{c}(t-\tau)} b_{c} d \tau+c A_{c}^{2} b_{c}\right|\left\|\eta_{\Delta}\right\|_{\infty}^{\prime} \\
& \quad+\left.\left|c A_{c} b_{c}\right||| \frac{d}{d t} u^{*}\right|_{\infty} ^{\prime}
\end{aligned}
$$

and

$$
\begin{aligned}
& \left|\zeta_{\Delta}^{(3)}\left(\left(k+1+\psi_{k+1}^{\Delta}\right) \Delta\right)-\zeta_{\Delta}^{(3)}\left(\left(k+\psi_{k}^{\Delta}\right) \Delta\right)\right| \\
& \quad \leq 2 \sup _{t \in\left[0, t_{f}\right]}\left|c A_{c}^{3} \int_{0}^{t} e^{A_{c}(t-\tau)} b_{c} d \tau+c A_{c}^{2} b_{c}\right|\left\|\eta_{\Delta}\right\|_{\infty}^{\prime} \\
& \quad+\left|c A_{c} b_{c}\right| \sup _{\left|t_{1}-t_{2}\right| \leq \Delta}\left|\frac{d}{d t} u^{*}\left(t_{1}\right)-\frac{d}{d t} u^{*}\left(t_{2}\right)\right| .
\end{aligned}
$$

Since $(d / d t) u^{*} \quad \in \quad C^{0}\left[0, t_{f}\right]$, we can see that $\left[\zeta_{\Delta}^{(3)}\left(\left(1+\psi_{1}^{\Delta}\right) \Delta\right) \Delta / 6 \cdots \zeta_{\Delta}^{(3)}\left(\left(N-1+\psi_{N-1}^{\Delta}\right) \Delta\right) \Delta / 6\right]$ has the same limiting property as $w_{\Delta} \Delta$.
Similarly, we $\quad$ can
$\left[\zeta_{\Delta}^{(3)}\left(\left(1-\phi_{1}^{\Delta}\right) \Delta\right) \Delta / 6 \cdots \zeta_{\Delta}^{(3)}\left(\left(N-1-\phi_{N-1}^{\Delta}\right) \Delta\right) \Delta / 6\right]$
does likewise.

that

\section{ACKNOWLEDGMENT}

The authors would like to thank T. Hagiwara and the anonymous reviewers for their comments and suggestions.

\section{REFERENCES}

[1] Y. Hayakawa, S. Hosoe, and M. Ito, "On the limiting zeros of sampled multivariable systems," Syst. Control Lett., vol. 2, no. 5, pp. 292-300, 1983.

[2] K. J.Åström, P. Hagander, and J. Sternby, "Zeros of sampled systems," Automatica, vol. 20, no. 1, pp. 31-38, 1984.

[3] K. M. Passino and P. J. Antsaklis, "Inverse stable sampled low-pass systems," Int. J. Control, vol. 47, no. 6, pp. 1905-1913, 1988.

[4] Y. Fu and G. A. Dumont, "Choice of sampling to ensure minimumphase behavior," IEEE Trans. Automat. Contr., vol. 34, pp. 560-563, May 1989.

[5] K. Diekmann, K. H. Fasol, S. Jayasuriya, and M. Rabins, "On the spurious zeros of discrete-transfer functions," in Proc. Amer. Control Conf., 1990, pp. 1162-1163.

[6] M. Ishitobi, "Stable zeros of sampled low-pass systems," Int. J. Control, vol. 57, no. 6, pp. 1485-1498, 1993.

[7] J. J. Chen and C.-H. Menq, "Design of bi-causal inverse models for nonminimum phase systems and its applications to precision tracking control," in Proc. Amer. Control Conf., 1996, pp. 3988-3993.

[8] T. Hagiwara, "Analytic study on the intrinsic zeros of sampled-data systems," IEEE Trans. Automat. Contr., vol. 41, pp. 261-263, Feb. 1996.

[9] M. J. Blachuta, "On zeros of sampled systems," IEEE Trans. Automat. Contr., vol. 44, pp. 1229-1234, June 1999.

[10] K. Fuwa, T. Narikiyo, and Y. Funahashi, "A construction of an inverse model with cut-off filter and its application to model feedback control system," Trans. Inst. Electr. Eng. Japan, vol. 119-C, pp. 1444-1453, 1999.

[11] K. L. Moore, Iterative Learning Control for Deterministic Systems. New York: Springer-Verlag, 1993.

[12] Z. Bien and J. X. Xu, Eds., Iterative Learning Control-Analysis, Design, Integration and Applications. Norwell, MA: Kluwer, 1998.

[13] T. Sogo and N. Adachi, "Convergence rates and robustness of iterative learning control," in Proc. 35th IEEE Conf. Decision Control, 1996, pp. $3050-3055$.

[14] — "On properties of limiting zeros of sampled-data systems on finite time domains and the inverse systems" (in in Japanese), Trans. Soc. Instrum. Control Eng., vol. 34, no. 10, pp. 1395-1403, 1998

[15] T. Hagiwara and M. Araki, "Stability of the limiting zeros of sampled-data systems with zero and first-order holds," Int. J. Control, vol. 58, no. 6, pp. 1325-1346, 1993. 